\title{
Comparison Results on Preconditioned GAOR Methods for Weighted Linear Least Squares Problems
}

\author{
Guangbin Wang, ${ }^{1}$ Yanli Du, ${ }^{1}$ and Fuping Tan ${ }^{2}$ \\ ${ }^{1}$ Department of Mathematics, Qingdao University of Science and Technology, Qingdao 266061, China \\ ${ }^{2}$ Department of Mathematics, Shanghai University, Shanghai 200444, China
}

Correspondence should be addressed to Guangbin Wang, wguangbin750828@sina.com

Received 18 July 2012; Accepted 26 August 2012

Academic Editor: Zhongxiao Jia

Copyright (c) 2012 Guangbin Wang et al. This is an open access article distributed under the Creative Commons Attribution License, which permits unrestricted use, distribution, and reproduction in any medium, provided the original work is properly cited.

We present preconditioned generalized accelerated overrelaxation methods for solving weighted linear least square problems. We compare the spectral radii of the iteration matrices of the preconditioned and the original methods. The comparison results show that the preconditioned GAOR methods converge faster than the GAOR method whenever the GAOR method is convergent. Finally, we give a numerical example to confirm our theoretical results.

\section{Introduction}

Consider the weighted linear least squares problem

$$
\min _{x \in R^{n}}(A x-b)^{T} W^{-1}(A x-b)
$$

where $W$ is the variance-covariance matrix. The problem has many scientific applications. A typical source is parameter estimation in mathematical modeling.

This problem has been discussed in many books and articles. In order to solve it, one has to solve a nonsingular linear system as

$$
H y=f
$$


where

$$
H=\left(\begin{array}{cc}
I-B_{1} & U \\
C & I-B_{2}
\end{array}\right)
$$

is an invertible matrix with

$$
B_{1}=\left(b_{i j}\right)_{p \times p^{\prime}} \quad B_{2}=\left(b_{i j}\right)_{(n-p) \times(n-p)^{\prime}} \quad C=\left(c_{i j}\right)_{(n-p) \times p^{\prime}} \quad U=\left(u_{i j}\right)_{p \times(n-p)^{\prime}} .
$$

Yuan proposed a generalized SOR (GSOR) method to solve linear system (1) in [1]; afterwards, Yuan and Jin [2] established a generalized AOR (GAOR) method to solve linear system (1). In $[3,4]$, authors studied the convergence of the GAOR method for solving the linear system $H y=f$. In [3], authors studied the convergence of the GAOR method when the coefficient matrices are consistently ordered matrices and gave the regions of convergence. In [4], authors studied the convergence of the GAOR method for diagonally dominant coefficient matrices and gave the regions of convergence.

In order to solve the linear system (1.2) using the GAOR method, we split $H$ as

$$
H=I-\left(\begin{array}{cc}
0 & 0 \\
-C & 0
\end{array}\right)-\left(\begin{array}{cc}
B_{1} & -U \\
0 & B_{2}
\end{array}\right)
$$

Then, for $\omega \neq 0$, one GAOR method can be defined by

$$
y^{(k+1)}=L_{r, \omega} y^{(k)}+\omega g, \quad k=0,1,2, \ldots,
$$

where

$$
\begin{aligned}
L_{r, \omega} & =\left(\begin{array}{cc}
I & 0 \\
r C & I
\end{array}\right)^{-1}\left[(1-\omega) I+(\omega-r)\left(\begin{array}{cc}
0 & 0 \\
-C & 0
\end{array}\right)+\omega\left(\begin{array}{cc}
B_{1} & -U \\
0 & B_{2}
\end{array}\right)\right] \\
& =\left(\begin{array}{cc}
(1-\omega) I+\omega B_{1} & -\omega U \\
\omega(r-1) C-\omega r C B_{1} & (1-\omega) I+\omega B_{2}+\omega r C U
\end{array}\right)
\end{aligned}
$$

is the iteration matrix and

$$
g=\left(\begin{array}{cc}
I & 0 \\
-r C & I
\end{array}\right) f
$$

In order to decrease the spectral radius of $L_{r, \omega}$, an effective method is to precondition the linear system (1.2), namely,

$$
P H=\left(\begin{array}{cc}
I-B_{1}^{*} & U^{*} \\
C^{*} & I-B_{2}^{*}
\end{array}\right)
$$


then the preconditioned GAOR method can be defined by

$$
y^{(k+1)}=L_{r, \omega}^{*} y^{(k)}+\omega g^{*}, \quad k=0,1,2, \ldots
$$

where

$$
\begin{gathered}
L_{r, \omega}^{*}=\left(\begin{array}{cc}
(1-\omega) I+\omega B_{1}^{*} & -\omega U^{*} \\
\omega(1-r) C^{*}-\omega r C^{*} B_{1}^{*} & (1-\omega) I+\omega B_{2}^{*}+\omega r C^{*} U^{*}
\end{array}\right), \\
g^{*}=\left(\begin{array}{cc}
I & 0 \\
-r C^{*} & I
\end{array}\right) P f .
\end{gathered}
$$

In [5], authors presented three kinds of preconditioners for preconditioned modified accelerated overrelaxation method to solve systems of linear equations. They showed that the convergence rate of the preconditioned modified accelerated overrelaxation method is better than that of the original method, whenever the original method is convergent.

This paper is organized as follows. In Section 2, we give some important definition and the known results as the preliminaries of the paper. In Section 3, we propose three preconditioners and give the comparison theorems between the preconditioned and original methods. These results show that the preconditioned GAOR methods converge faster than the GAOR method whenever the GAOR method is convergent. In Section 4, we give an example to confirm our theoretical results.

\section{Preliminaries}

We need the following definition and results.

Definition 2.1. Let $A=\left(a_{i j}\right)_{n \times n}$ and $B=\left(b_{i j}\right)_{n \times n}$. We say $A \geq B$ if $a_{i j} \geq b_{i j}$ for all $i, j=1,2, \ldots, n$. This definition can be carried over to vectors by identifying them with $n \times 1$ matrices. In this paper, $\rho(\cdot)$ denotes the spectral radius of a matrix.

Lemma 2.2 (see [6]). Let $A \in R^{n \times n}$ be nonnegative and irreducible. Then

(i) $A$ has a positive real eigenvalue equal to its spectral radius $\rho(A)$;

(ii) for $\rho(A)$, there corresponds an eigenvector $x>0$.

Lemma 2.3 (see [7]). Let $A \in R^{n \times n}$ be nonnegative and irreducible. If

$$
0 \neq \alpha x \leq A x \leq \beta x, \quad \alpha x \neq A x, \quad A x \neq \beta x,
$$

for some nonnegative vector $x$, then $\alpha<\rho(A)<\beta$ and $x$ is a positive vector.

\section{Comparison Results}

We consider the preconditioned linear system

$$
\widetilde{H} y=\tilde{f},
$$


where $\widetilde{H}=(I+\widetilde{S}) H$ and $\tilde{f}=(I+\widetilde{S}) f$ with

$$
\widetilde{S}=\left(\begin{array}{ll}
S & 0 \\
0 & 0
\end{array}\right)
$$

$S$ is a $p \times p$ matrix with $1<p<n$.

We take $S$ as follows:

$$
S_{1}=\left(\begin{array}{ccccc}
0 & \alpha_{2} b_{12} & \cdots & 0 & 0 \\
\beta_{2} b_{21} & 0 & \ddots & 0 & 0 \\
\vdots & \ddots & \vdots & \ddots & \vdots \\
0 & 0 & \ddots & 0 & \alpha_{p} b_{p-1, p} \\
0 & 0 & \cdots & \beta_{p} b_{p, p-1} & 0
\end{array}\right), \quad S_{2}=\left(\begin{array}{cccc}
0 & \alpha_{2} b_{12} & \cdots & \alpha_{p} b_{1 p} \\
\beta_{2} b_{21} & 0 & \cdots & 0 \\
\vdots & \vdots & \ddots & \vdots \\
\beta_{p} b_{p 1} & 0 & \cdots & 0
\end{array}\right)
$$

Now, we obtain two preconditioned linear systems with coefficient matrices

$$
\widetilde{H}_{i}=\left(\begin{array}{cc}
I-\left[B_{1}-S_{i}\left(I-B_{1}\right)\right] & \left(I+S_{i}\right) U \\
C & I-B_{2}
\end{array}\right), \quad \text { for } i=1,2
$$

where

$$
\begin{aligned}
& B_{1}-S_{1}\left(I-B_{1}\right) \\
& =\left(\begin{array}{ccc}
b_{11}+\alpha_{2} b_{21} b_{12} & \cdots & b_{1 p}+\alpha_{2} b_{2 p} b_{12} \\
b_{21}+\alpha_{3} b_{23} b_{31}-\beta_{2} b_{21}\left(1-b_{11}\right) & \cdots & b_{2 p}+\beta_{2} b_{1 p} b_{21}+\alpha_{3} b_{3 p} b_{23} \\
\vdots & \vdots & \vdots \\
b_{p-1,1}+\beta_{p-1} b_{p-1, p-2} b_{p-2,1}+\alpha_{p} b_{p-1, p} b_{p 1} & \cdots & b_{p-1, p}+\beta_{p-1} b_{p-1, p-2} b_{p-2, p}+\alpha_{p} b_{p-2, p} b_{p-1, p-2} \\
b_{p 1}+\beta_{p} b_{p-1,1} b_{p, p-1} & \cdots & b_{p p}+\beta_{p} b_{p, p-1} b_{p-1, p}
\end{array}\right), \\
& B_{1}-S_{2}\left(I-B_{1}\right) \\
& =\left(\begin{array}{ccc}
b_{11}+\alpha_{2} b_{12} b_{21}+\cdots+\alpha_{p} b_{1 p} b_{p 1} & \cdots & b_{1 p}+\alpha_{2} b_{12} b_{2 p}+\cdots+\alpha_{p} b_{1 p}\left(1-b_{p p}\right) \\
b_{21}-\beta_{2} b_{21}\left(1-b_{11}\right) & \cdots & b_{2 p}+\beta_{2} b_{21} b_{1 p} \\
\vdots & \ddots & \vdots \\
b_{p 1}-\beta_{p} b_{p 1}\left(1-b_{11}\right) & \cdots & b_{p p}+\beta_{p} b_{p 1} b_{1 p}
\end{array}\right) .
\end{aligned}
$$

We split $\widetilde{H}_{i}(i=1,2)$ as

$$
\widetilde{H}_{i}=I-\left(\begin{array}{cc}
0 & 0 \\
-C & 0
\end{array}\right)-\left(\begin{array}{cc}
{\left[B_{1}-S_{i}\left(I-B_{1}\right)\right]} & -\left(I+S_{i}\right) U \\
0 & B_{2}
\end{array}\right), \quad \text { for } i=1,2,
$$


then the preconditioned GAOR methods for solving (3.1) are defined as follows

$$
y^{(k+1)}=L_{r, \omega}^{(i)} y^{(k)}+\omega \tilde{g}, \quad k=0,1,2, \ldots
$$

where

$$
L_{r, \omega}^{(i)}=\left(\begin{array}{cc}
(1-\omega) I+\omega\left[B_{1}-S_{i}\left(I-B_{1}\right)\right] & -\omega\left(I+S_{i}\right) U \\
\omega(r-1) C-\omega r C\left[B_{1}-S_{i}\left(I-B_{1}\right)\right] & (1-\omega) I+\omega B_{2}+\omega r C\left(I+S_{i}\right) U
\end{array}\right)
$$

are iteration matrices and

$$
\tilde{g}=\left(\begin{array}{cc}
I & 0 \\
-r C & I
\end{array}\right) \tilde{f}
$$

Now, we give comparison results between the preconditioned GAOR methods defined by (3.7) and the corresponding GAOR method defined by (1.6).

Theorem 3.1. Let $L_{r, \omega}, L_{r, \omega}^{(1)}$ be the iteration matrices associated with the GAOR and preconditioned $G A O R$ methods, respectively. If the matrix $H$ in (1.2) is irreducible with $C \leq 0, U \leq 0, B_{1} \geq 0, B_{2} \geq 0$, $0<\omega \leq 1,0 \leq r<1, b_{i, i+1}>0, b_{i+1, i}>0$ for some $i \in\{2, \ldots, p\}$, when $0 \leq b_{i i}<1(i \in\{2, \ldots, p\})$,

$$
\begin{array}{cl}
0<\alpha_{i}<\frac{b_{i-1, i-2} b_{i-2, i}+b_{i-1, i}\left(1-b_{i-2, i-2}\right)}{b_{i-1, i-2}\left[\left(1-b_{i i}\right)\left(1-b_{i-2, i-2}\right)-b_{i, i-2} b_{i-2, i}\right]} & \text { for } i \in\{3, \ldots, p\}, \quad \alpha_{2}<\frac{1}{1-b_{22}}, \\
0<\beta_{i}<\frac{b_{i, i-1}\left(1-b_{i+1, i+1}\right)+b_{i-1, i} b_{i+1, i-1}}{b_{i, i-1}\left[\left(1-b_{i-1, i-1}\right)\left(1-b_{i+1, i+1}\right)-b_{i-1, i+1} b_{i+1, i-1}\right]} & \text { for } i \in\{2, \ldots, p-1\}, \quad \beta_{p}<\frac{1}{1-b_{p p}},
\end{array}
$$

or when $b_{i i} \geq 1, \alpha_{i}>0, \beta_{i}>0(i \in\{2, \ldots, p\})$, then either

$$
\rho\left(L_{r, \omega}^{(1)}\right)<\rho\left(L_{r, \omega}\right)<1
$$

or

$$
\rho\left(L_{r, \omega}^{(1)}\right)>\rho\left(L_{r, \omega}\right)>1
$$

Proof. By direct operation, we have

$$
L_{r, \omega}=\left(\begin{array}{cc}
(1-\omega) I+\omega B_{1} & -\omega U \\
-\omega(1-r) C & (1-\omega) I+\omega B_{2}
\end{array}\right)+\omega r\left(\begin{array}{cc}
0 & 0 \\
-C B_{1} & C U
\end{array}\right) .
$$


Since $0<\omega \leq 1,0 \leq r<1, C \leq 0, U \leq 0, B_{1} \geq 0, B_{2} \geq 0$, then

$$
\left(\begin{array}{cc}
0 & 0 \\
-C B_{1} & C U
\end{array}\right) \geq 0
$$

and $L_{r, \omega}$ is nonnegative. Since $H$ is irreducible, from (3.13), it is easy to see that the matrix $L_{r, \omega}$ is nonnegative and irreducible.

Similarly, we can prove that the matrix $L_{r, \omega}^{(1)}$ is a nonnegative and irreducible matrix.

By Lemma 2.2, there is a positive vector $x$ such that

$$
L_{r, \omega} x=\lambda x
$$

where $\lambda=\rho\left(L_{r, \omega}\right)$. Since the matrix $H$ is nonsingular, $\lambda \neq 1$. Hence, we get either $\lambda>1$ or $\lambda<1$.

Now, from (3.15) and by the definitions of $L_{r, \omega}$ and $L_{r, \omega}^{(1)}$, we have

$$
\begin{aligned}
& L_{r, \omega}^{(1)} x-\lambda x=\left(L_{r, \omega}^{(1)}-L_{r, \omega}\right) x \\
& =\left(\begin{array}{cc}
-\omega S_{1}\left(I-B_{1}\right) & -\omega S_{1} U \\
\omega r C S_{1}\left(I-B_{1}\right) & \omega r C S_{1} U
\end{array}\right) x \\
& =\left(\begin{array}{cc}
S_{1} & 0 \\
-r C S_{1} & 0
\end{array}\right)\left(\begin{array}{cc}
-\omega\left(I-B_{1}\right) & -\omega U \\
0 & 0
\end{array}\right) x \\
& =\left(\begin{array}{cc}
S_{1} & 0 \\
-r C S_{1} & 0
\end{array}\right)\left(\begin{array}{cc}
-\omega\left(I-B_{1}\right) & -\omega U \\
\omega(r-1) C-\omega r C B_{1} & -\omega I+\omega B_{2}+\omega r C U
\end{array}\right) x \\
& =\left(\begin{array}{cc}
S_{1} & 0 \\
-r C S_{1} & 0
\end{array}\right)\left(L_{r, \omega}-I\right) x=(\lambda-1)\left(\begin{array}{cc}
S_{1} & 0 \\
-r C S_{1} & 0
\end{array}\right) x .
\end{aligned}
$$

Since $b_{i, i+1}>0, b_{i+1, i}>0$ for some $i \in\{2, \ldots, p\}$, when $0 \leq b_{i i}<1(i \in\{2, \ldots, p\})$,

$$
\begin{gathered}
0<\alpha_{i}<\frac{b_{i-1, i-2} b_{i-2, i}+b_{i-1, i}\left(1-b_{i-2, i-2}\right)}{b_{i-1, i-2}\left[\left(1-b_{i i}\right)\left(1-b_{i-2, i-2}\right)-b_{i, i-2} b_{i-2, i}\right]} \quad \text { for } i \in\{3, \ldots, p\}, \quad \alpha_{2}<\frac{1}{1-b_{22}}, \\
0<\beta_{i}<\frac{b_{i, i-1}\left(1-b_{i+1, i+1}\right)+b_{i-1, i} b_{i+1, i-1}}{b_{i, i-1}\left[\left(1-b_{i-1, i-1}\right)\left(1-b_{i+1, i+1}\right)-b_{i-1, i+1} b_{i+1, i-1}\right]} \quad \text { for } i \in\{2, \ldots, p-1\}, \quad \beta_{p}<\frac{1}{1-b_{p p}},
\end{gathered}
$$


or when $b_{i i} \geq 1, \alpha_{i}>0, \beta_{i}>0(i \in\{2, \ldots, p\})$, then $S_{1} \geq 0$ and $S_{1} \neq 0$. So we have $\left(\begin{array}{cc}S_{1} & 0 \\ -r C S_{1} & 0\end{array}\right) x \geq$ $0,\left(\begin{array}{cc}S_{1} & 0 \\ -r C S_{1} & 0\end{array}\right) x \neq 0$.

If $\lambda<1$, then $L_{r, \omega}^{(1)} x-\lambda x \leq 0, L_{r, \omega}^{(1)} x-\lambda x \neq 0$.

By Lemma 2.3, the inequality (3.11) is proved.

If $\lambda>1$, then $L_{r, \omega}^{(1)} x-\lambda x \geq 0, L_{r, \omega}^{(1)} x-\lambda x \neq 0$.

By Lemma 2.3, the inequality (3.12) is proved.

Theorem 3.2. Let $L_{r, \omega}, L_{r, \omega}^{(2)}$ be the iteration matrices associated with the GAOR and preconditioned GAOR methods, respectively. If the matrix $H$ in (1.2) is irreducible with $C \leq 0, U \leq 0, B_{1} \geq 0$, $B_{2} \geq 0,0<\omega \leq 1,0 \leq r<1, b_{i, 1}>0, b_{1, i}>0$ for some $i \in\{2,3, \ldots, p\}$, when $0 \leq b_{11}<1$, $0<\beta_{i}<1 /\left(1-b_{11}\right)$,

$$
0<\alpha_{i}<\frac{b_{1 i}+\alpha_{2} b_{12} b_{2 i}+\cdots \alpha_{i-1} b_{1, i-1} b_{i-1, i}+\alpha_{i+1} b_{1, i+1} b_{i+1, i}+\cdots+\alpha_{p} b_{1 p} b_{p i}}{b_{1 i}\left(1-b_{i i}\right)}, \quad i \in\{2,3, \ldots, p\}
$$

or when $b_{11} \geq 1, \alpha_{i}>0, \beta_{i}>0, i \in\{2,3, \ldots, p\}$, then either

$$
\rho\left(L_{r, \omega}^{(2)}\right)<\rho\left(L_{r, \omega}\right)<1
$$

or

$$
\rho\left(L_{r, \omega}^{(2)}\right)>\rho\left(L_{r, \omega}\right)>1
$$

By the analogous proof of Theorem 3.1, we can prove Theorem 3.2.

\section{Numerical Example}

Now, we present an example to illustrate our theoretical results.

Example 4.1. The coefficient matrix $H$ in (1.2) is given by

$$
H=\left(\begin{array}{cc}
I-B_{1} & U \\
C & I-B_{2}
\end{array}\right)
$$


Table 1: The spectral radii of the GAOR and preconditioned GAOR iteration matrices.

\begin{tabular}{lcccccc}
\hline$n$ & $\omega$ & $r$ & $p$ & $\rho$ & $\rho_{1}$ & $\rho_{2}$ \\
\hline 5 & 0.95 & 0.7 & 3 & 0.1450 & 0.1384 & 0.1348 \\
10 & 0.9 & 0.85 & 5 & 0.2782 & 0.2726 & 0.2695 \\
15 & 0.95 & 0.8 & 5 & 0.3834 & 0.3808 & 0.3796 \\
20 & 0.75 & 0.65 & 10 & 0.6350 & 0.6317 & 0.6297 \\
25 & 0.7 & 0.55 & 8 & 0.7872 & 0.7861 & 0.7855 \\
30 & 0.65 & 0.55 & 16 & 0.9145 & 0.9136 & 0.9130 \\
40 & 0.6 & 0.5 & 10 & 1.1426 & 1.1433 & 1.1436 \\
50 & 0.6 & 0.5 & 10 & 1.3668 & 1.3683 & 1.3691 \\
\hline
\end{tabular}

where $B_{1}=\left(b_{i j}^{(1)}\right)_{p \times p^{\prime}}, B_{2}=\left(b_{i j}^{(2)}\right)_{(n-p) \times(n-p)^{\prime}} C=\left(c_{i j}\right)_{(n-p) \times p^{\prime}}$ and $U=\left(u_{i j}\right)_{p \times(n-p)}$ with

$$
\begin{aligned}
& b_{i i}^{(1)}=\frac{1}{10 \times(i+1)}, \quad i=1,2, \ldots, p, \\
& b_{i j}^{(1)}=\frac{1}{30}-\frac{1}{30 \times j+i^{\prime}}, \quad i<j, i=1,2, \ldots, p-1, j=2, \ldots, p, \\
& b_{i j}^{(1)}=\frac{1}{30}-\frac{1}{30 \times(i-j+1)+i}, \quad i>j, i=2, \ldots, p, j=1,2, \ldots, p-1, \\
& b_{i i}^{(2)}=\frac{1}{10 \times(p+i+1)}, \quad i=1,2, \ldots, n-p, \\
& b_{i j}^{(2)}=\frac{1}{30}-\frac{1}{30 \times(p+j)+p+i}, \quad i<j, i=1,2, \ldots, n-p+1, j=2, \ldots, n-p, \\
& b_{i j}^{(2)}=\frac{1}{30}-\frac{1}{30 \times(i-j+1)+p+i}, \quad i>j, i=, 2, \ldots, n-p, j=1,2, \ldots, n-p-1, \\
& c_{i j}=\frac{1}{30 \times(p+i-j+1)+p+i}-\frac{1}{30}, \quad i=1,2, \ldots, n-p, j=1,2, \ldots, p, \\
& u_{i j}=\frac{1}{30 \times(p+j)+i}-\frac{1}{30}, \quad i=1,2, \ldots, p, j=1,2, \ldots, n-p .
\end{aligned}
$$

Table 1 displays the spectral radii of the corresponding iteration matrices with some randomly chosen parameters $r, \omega, p$. The randomly chosen parameters $\alpha_{i}$ and $\beta_{i}$ satisfy the conditions of two theorems.

From Table 1, we see that these results accord with Theorems 3.1-3.2.

\section{Acknowledgments}

This work was supported by the National Natural Science Foundation of China (Grant no. 11001144) and the Science and Technology Program of Shandong Universities of China (J10LA06). 


\section{References}

[1] J.-Y. Yuan, "Numerical methods for generalized least squares problems," Journal of Computational and Applied Mathematics, vol. 66, no. 1-2, pp. 571-584, 1996.

[2] J.-Y. Yuan and X.-Q. Jin, "Convergence of the generalized AOR method," Applied Mathematics and Computation, vol. 99, no. 1, pp. 35-46, 1999.

[3] M. T. Darvishi, P. Hessari, and J. Y. Yuan, "On convergence of the generalized accelerated overrelaxation method," Applied Mathematics and Computation, vol. 181, no. 1, pp. 468-477, 2006.

[4] M. T. Darvishi and P. Hessari, "On convergence of the generalized AOR method for linear systems with diagonally dominant coefficient matrices," Applied Mathematics and Computation, vol. 176, no. 1, pp. 128-133, 2006.

[5] M. T. Darvishi, P. Hessari, and B.-C. Shin, "Preconditioned modified AOR method for systems of linear equations," International Journal for Numerical Methods in Biomedical Engineering, vol. 27, no. 5, pp. 758$769,2011$.

[6] R. S. Varga, Matrix Iterative Analysis, vol. 27 of Springer Series in Computational Mathematics, Springer, Berlin, Germany, 2000.

[7] A. Berman and R. J. Plemmons, Nonnegative Matrices in the Mathematical Sciences, vol. 9 of Classics in Applied Mathematics, SIAM, Philadelphia, Pa, USA, 1994. 


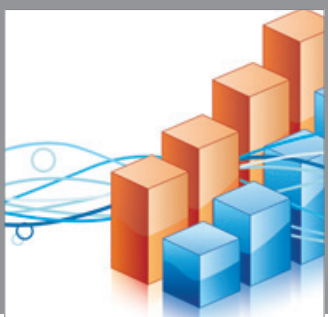

Advances in

Operations Research

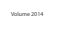

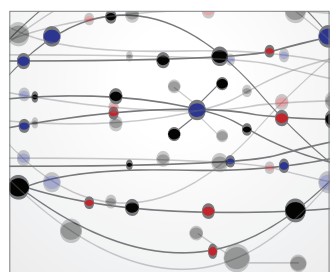

\section{The Scientific} World Journal
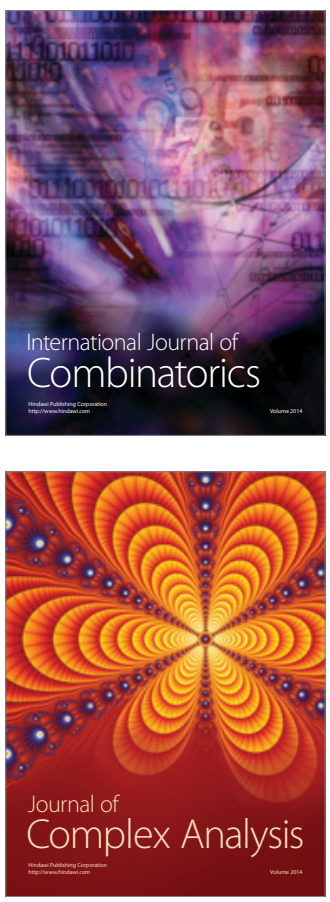

International Journal of

Mathematics and

Mathematical

Sciences
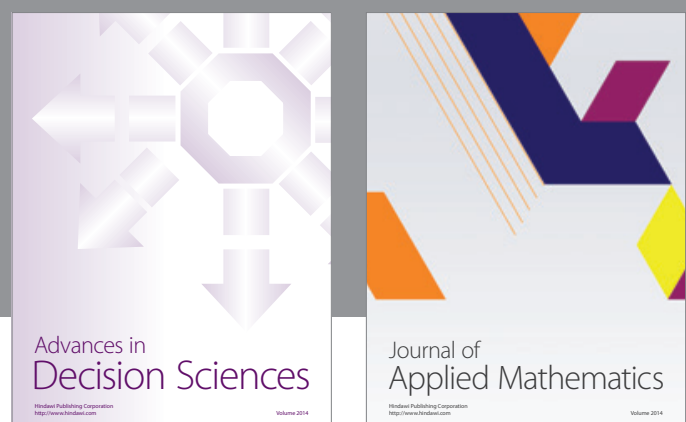

Journal of

Applied Mathematics
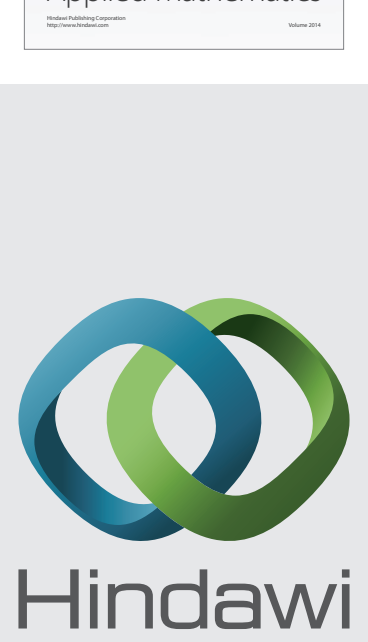

Submit your manuscripts at http://www.hindawi.com
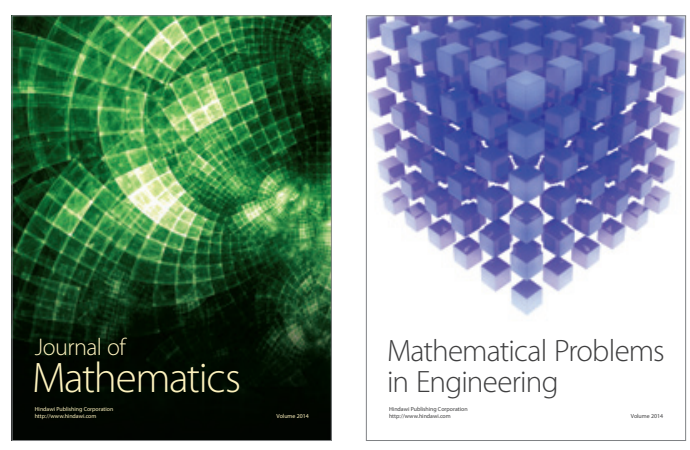

Mathematical Problems in Engineering
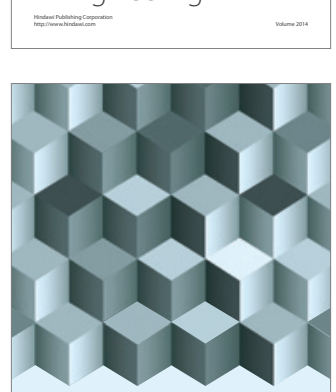

Journal of

Function Spaces
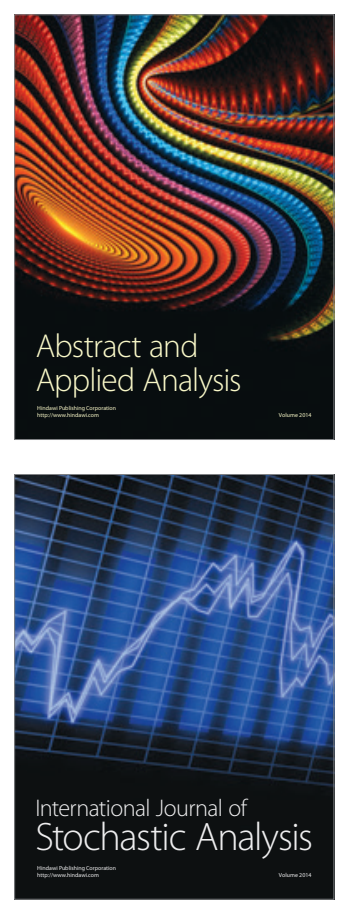

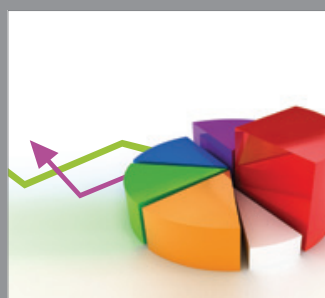

ournal of

Probability and Statistics

Promensencen
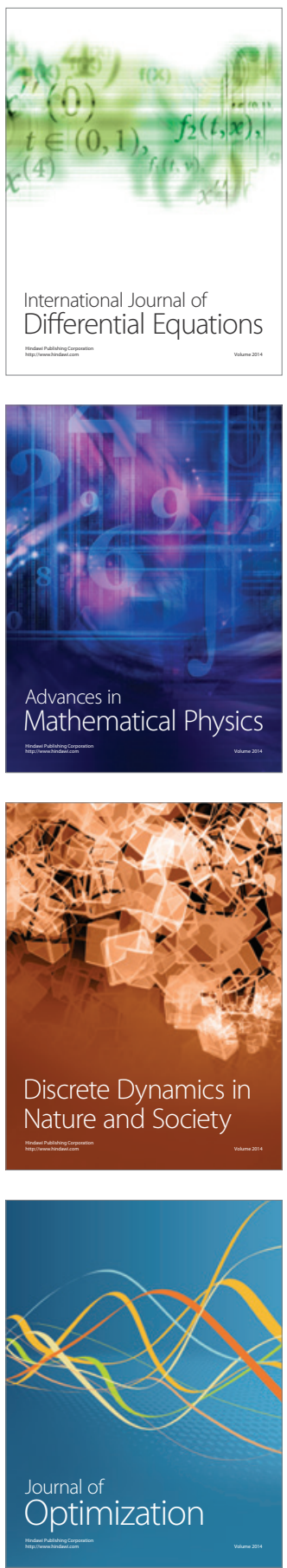